\title{
The impact of COVID-19 on primary health care and antibiotic prescribing in rural China: qualitative study
}

Tingting Zhang ${ }^{1 *}$, Xingrong Shen ${ }^{2}$, Rong Liu ${ }^{2}$, Linhai Zhao ${ }^{2}$, Debin Wang ${ }^{2}$, Helen Lambert ${ }^{1}$ and Christie Cabral $^{3}$

\begin{abstract}
Background: In China, the primary health care (PHC) system has been designated responsible for control and prevention of COVID-19, but not treatment. Suspected COVID-19 cases presenting to PHC facilities must be transferred to specialist fever clinics. This study aims to understand the impact of COVID-19 on PHC delivery and on antibiotic prescribing at a community level in rural areas of central China.

Methods: Qualitative semi-structured interviews were conducted with 18 PHC practitioners and seven patients recruited from two township health centres and nine village clinics in two rural residential areas of Anhui province. Interviews were transcribed verbatim and analysed thematically.

Results: PHC practitioners reported a major shift in their work away from seeing and treating patients (due to government-mandated referral to specialist Covid clinics) to focus on the key public health roles of tracing, screening and educating in rural areas. The additional work, risk, and financial pressure that PHC practitioners faced, placed considerable strain on them, particularly those working in village clinics. Face to face PHC provision was reduced and there was no substitution with consultations by phone or app, which practitioners attributed to the fact that most of their patients were elderly and not willing or able to switch. Practitioners saw COVID-19 as outside of their area of expertise and very different to the non-COVID-19 respiratory tract infections that they frequently treated pre-pandemic. They reported that antibiotic prescribing was reduced overall because far fewer patients were attending rural PHC facilities, but otherwise their antibiotic prescribing practices remained unchanged.

Conclusions: The COVID-19 pandemic had considerable impact on PHC in rural China. Practitioners took on substantial additional workload as part of epidemic control and fewer patients were seen in PHC. The reduction in patients seen and treated in PHC led to a reduction in antibiotic prescribing, although clinical practice remains unchanged. Since COVID-19 epidemic control work has been designated as a long-term task in China, rural PHC clinics now face the challenge of how to balance their principal clinical and increased public health roles and, in the case of the village clinics, remain financially viable.
\end{abstract}

Keywords: COVID-19, antibiotic treatment, epidemic prevention, primary health care delivery, workload, financial pressure

\footnotetext{
* Correspondence: tingting.zhang@bristol.ac.uk

'Population Health Sciences, Bristol Medical School, University of Bristol, Bristol, UK

Full list of author information is available at the end of the article
}

(C) The Author(s). 2021 Open Access This article is licensed under a Creative Commons Attribution 4.0 International License, which permits use, sharing, adaptation, distribution and reproduction in any medium or format, as long as you give appropriate credit to the original author(s) and the source, provide a link to the Creative Commons licence, and indicate if changes were made. The images or other third party material in this article are included in the article's Creative Commons licence, unless indicated otherwise in a credit line to the material. If material is not included in the article's Creative Commons licence and your intended use is not permitted by statutory regulation or exceeds the permitted use, you will need to obtain permission directly from the copyright holder. To view a copy of this licence, visit http://creativecommons.org/licenses/by/4.0/ The Creative Commons Public Domain Dedication waiver (http://creativecommons.org/publicdomain/zero/1.0/) applies to the data made available in this article, unless otherwise stated in a credit line to the data. 


\section{Background}

The aggressive COVID-19 pandemic has stressed health systems around the world. Primary health care (PHC) systems in countries like the UK and Australia have rapidly responded to COVID-19, including key measures like shifting to remote consultations and providing online training and guidance, whilst facing challenges to manage increased workload and to practice good infection control [1, 2]. In China, PHC practitioners were allocated responsibility for conducting COVID-19 screening and referral, monitoring, education and publicity by the National Health Commission [3]. The regulations and guidance issued by state governments strictly prohibit any consultation or treatment for patients with fever or cough in $\mathrm{PHC}$ and require all suspected COVID-19 patients to be transferred to specialist fever clinics at designated centres [4]. Evidence from a nationwide study showed that over $90 \%$ of PHC institutions have implemented these new tasks [5]. Local case studies have also demonstrated the key role of $\mathrm{PHC}$ practitioners in monitoring suspected COVID-19 cases at community level [6, 7], including identifying migrant workers returned from high-risk areas and managing their home quarantine over the Spring Festival [8].

$\mathrm{PHC}$ is a relatively weak but rapidly growing sector of China's health care system. Urban PHC services comprise community health centres and community health stations. Rural PHC services comprise township health centres (THCs) and, one level below them, village clinics (VCs). PHC provides general medical services and basic public health services to residents at community level. Since 2009, community health centres, THCs and VCs were designated responsible for delivering a package of essential public health services to all residents in urban and rural China. The main services included in the package are establishment resident health records, child, elderly and maternal health management, management of several chronic diseases, handling of communicable diseases, vaccination and health education [9]. Traditional Chinese Medicine (TCM) is also widely provided within PHC, with over $90 \%$ of community health centres and THCs delivering TCM along with general biomedical services in 2016; TCM health management is an important part of public health services in PHC [10]. China's PHC system differs in important ways from that in highincome settings. VCs offer only outpatient care and are staffed by 'village doctors' ${ }^{2}$, most of whom do not hold a medical degree and have varied medical training, whereas THCs have inpatient wards and a range of

\footnotetext{
2 'Village doctor' is a title that the Chinese government developed in 1985 to replace 'barefoot doctor'. 'Village doctors' only required have a technical school education or more than 20 years' practising experience in village clinics, and they are approved by local authorities to work in village clinics only with a 'village doctor certificate', rather than a regular license [11].
}

outpatient clinics staffed by practitioners who have received some formal medical training [12]. To accommodate the increasing demand for health care from the rural population, 'village doctors' in VCs are allowed to diagnose and treat patients attending for general illness such as infections, to prescribe medicines from the $\mathrm{Na}$ tional Essential Medicine List, and to refer patients to THCs or higher-level hospitals [11, 13]. Most VCs are independent entities that are non-government owned and financially semi-autonomous, charging fees that are either paid by patients or reclaimed from insurance $[9$, 14]. The THCs deliver technical guidance to VCs and have oversight of the public health work conducted by VC staff [12]. These frontline health institutions are most rural residents' first choice when seeking outpatient care [9]; in rural areas, $64.4 \%$ of patients chose to visit $\mathrm{VCs}$ and $22.4 \%$ visit $\mathrm{THCs}$ for general illness [15]. However, there are shortfalls in the quality of PHC, particularly in rural areas [5].

Antibiotics are probably over prescribed in rural PHC. The average proportion of antibiotics used for outpatient encounters in PHC institutions was over $50 \%$ [16], which is much higher than the percentage use of antibiotics recommended by the WHO $(\leq 30 \%)$ [17]. In clinical consultations, infection (ganran) is usually described as 'inflammation' (yan), a term that carries connotations of the redness and heat often seen with infections, and which is more readily understood by patients. Antibiotics are commonly called 'anti-inflammatory medicine' (xiaoyan yao) by practitioners and patients, and frequently prescribed for infectious illness or 'inflammation' (yan) [14]. One study conducted in the same area of China as the present study indicated that, in THCs and VCs, nearly $90 \%$ of patients with a respiratory tract infection (RTI) or urinary tract infection (UTI) were prescribed antibiotics [14].

PHC systems around the world are at risk of being overwhelmed by the COVID-19 pandemic [18]. Primary care practitioners in China are experiencing physical and mental pressures as a result of the pandemic, that impact their ability to work efficiently [19]. There has been very little research into the experiences of practitioners in rural $\mathrm{PHC}$, and $\mathrm{VCs}$ in particular, or into how COVID-19 affects antibiotic use in rural PHC. With the outbreak of COVID-19 and the introduction of new epidemic control regulations and measures, we undertook a qualitative study to investigate the impact of COVID-19 on PHC delivery and antibiotic prescribing at community level in the rural areas of central China.

\section{Methods}

Study area and recruitment methods

This study was carried out in two rural residential areas of Anhui province, one in Huangshan municipality and 
one in Fuyang municipality. Anhui province is located in the northeast of central China and more than half of Anhui's population lives in rural areas [20]. Since the beginning of March, the rate of new COVID-19 positive cases in Anhui province has remained stable; as of the 25th November 2020, there have been 992 positive cases. Fuyang has the 3rd highest incidence of all 16 municipalities of Anhui province, with 156 positive cases. Huangshan is a low incidence area in Anhui and only has 9 positive cases confirmed [21].

PHC practitioners that had participated in the codesign of an intervention for an ongoing trial were invited to participate in this study [22]. Participants were purposively recruited to capture a range of situations, including township and village, larger and smaller clinics, TCM and Western Medicine practitioners, and different geographical and COVID-19 incidence areas in Anhui province. Access to participants was secured via seniorlevel staff at the two selected THCs, who were identified through the existing networks of the research team. Practitioners were contacted or introduced by the senior staff and all agreed to interview. The practitioners then identified a small number of patients, all of whom agreed to be interviewed. In Chinese society with a strong culture of conformity, anonymous and formal approaches to study recruitment are often treated with suspicion and likely to cause people to either refuse to participate or to provide a socially acceptable account, whereas trust is engendered by contacts made through personal relationships [23-25]. Our recruitment method was therefore adapted to the cultural context of rural China to avoid low response rates and social desirability biases [24, 26]. Practitioners were recruited from one THC and five VCs in Huangshan municipality, and one THC and four VCs in Fuyang municipality. Because of the substantial decrease in patients in VCs due to the epidemic control measures, all patients were recruited from the two THCs.

This study was reviewed and approved by the Ethics Committee of Anhui Medical University (reference number 2020H0110). All methods were performed in accordance with the relevant guidelines and regulations. Audio recorded verbal consent was obtained from all participants and this method for recording consent was approved by the Ethics Committee.

\section{Data collection}

Qualitative methods enable detailed investigation of participants' views and experiences and are well-suited to studies that explore new phenomenon and focus on specific settings $[27,28]$. This approach was therefore used to investigate how participants understand the impact of COVID-19 on $\mathrm{PHC}$ and antibiotic prescribing at rural community level. Semi-structured interviews in Mandarin were conducted by a bilingual researcher (TZ), who has experience of conducting multiple qualitative interview studies, including several with rural health professionals and patients in rural China. Participants were first contacted by the researcher to confirm the interview time. Interviews were then conducted by phone or WeChat, in line with participant preference, and recorded using an encrypted audio-recorder. Interviews were conducted between 15th July and 2nd October 2020. The interviews ranged in length from 14 to $45 \mathrm{~min}$. Following standard qualitative methods, the sample size was determined using thematic saturation: transcripts were analysed iteratively to identify codes and themes and scheduling of new interviews stopped when no new codes or themes emerged from the data [29].

The semi-structured interviews were guided by topic guides, one for heads of PHC institutions, one for practitioners, and one for patients (see supplemental files). The interviews explored views and experiences of the impact of COVID-19 on PHC delivery. The topic guides covered: impacts on PHC delivery and health care seeking practices; experiences of and understandings of COVID-19; and how the COVID-19 pandemic had affected the use of antibiotics.

\section{Data analysis}

The thematic analysis approach enables coding and analysing of qualitative data to be undertaken in a systematic way that provides a robust analysis and enables links to be made to broader social theory [30]. All interviews were transcribed verbatim and analysed thematically in NVivo 12 Pro. A sub-sample of transcripts that captured the differences in participants' views and experiences were translated into English. Two researchers (TZ \& $\mathrm{CC}$ ) independently read these bilingual transcripts and assigned initial codes to the data; views and practices that reoccurred within and across participants were allocated into conceptual categories and themes. Researchers then worked collaboratively to develop the initial coding framework. The remaining transcripts were indexed by TZ using the initial coding framework and refinements were made as necessary. Practitioner and patient interviews were analysed separately. Common codes and categories emerged across transcripts of participants and developed into the major themes.

\section{Results}

Our sample consisted of 25 participants, including two township heads and seven township practitioners from two THCs, nine village practitioners, one from each of the nine VCs, together with seven patients from the two THCs. Among 18 practitioners, most (11) were general practitioners, two were TCM practitioners, two were internal medicine physicians, two were heads of THCs and 
one was a practitioner in outpatient department of the THC. Table 1 presents participant characteristics.

The results representing practitioners' and patients' views and perspectives on COVID-19 impacts on PHC services and the use of antibiotics are organised into five broad themes: (i) switch from PHC to epidemic prevention and control, which describes tracing and screening people, the decrease in patients but increase in workload, and awareness of COVID-19 management; (ii) concerns and challenges faced by those delivering PHC, including fear of COVID-19, physical and mental pressures in work, and financial pressures in VCs; (iii) diminished $\mathrm{PHC}$ and antibiotic prescribing, which covers the decreases in PHC delivery and access to and use of antibiotics in THCs and VCs; iv) COVID-19 as a different class of illness, and (v) the impact of COVID-19 on understandings of antibiotics use, including practitioners' and patients' antibiotic-related views and practices.

\section{Switch from primary health care to epidemic prevention and control}

Practitioners reported a major change in their work to focus on COVID-19 control and prevention and away from seeing and treating patients. Clinical services were largely suspended at the beginning of the outbreak and practitioners found the majority of their workload comprised tracing and screening people at high risk for
COVID-19: 'our main work is screening and seeing if there are any people who had fever. People who stay at local would be fine, we strictly screen people who returned from Wuhan or returned from Hubei' [P14, township internal medicine physician].

'During the epidemic prevention and control period, including pharmacies, telephone follow-up, and the tracing and follow-up of fever patients, there were more things to do and they become more complicated and fragmental. Other workload reduced, the main work is related to epidemic prevention and control.' [P1, head of THC]

Practitioners, particularly those working in VCs, described being allocated the additional work of reporting and tracing patients with fever identified in their clinics and delivering COVID-19 prevention information to residents in their area: 'we also need to ensure the prevention and publicity work for local population.' [P10, village practitioner].

Before the pandemic, THCs and VCs would diagnose and treat many patients presenting with respiratory infections and fever but after the introduction of epidemic control regulation, they were only allowed to screen and refer patients with suspected symptoms of COVID-19. Practitioners reported a large decrease in patients, particularly in VCs, attributed mainly to the diversion of

Table.1 Participant characteristics

\begin{tabular}{|c|c|c|c|}
\hline & & Practitioner participants & Patient participants \\
\hline Total number & & 18 & 7 \\
\hline \multirow[t]{2}{*}{ Gender } & Female & 2 & 5 \\
\hline & Male & 16 & 2 \\
\hline \multirow[t]{3}{*}{ Age } & $30-39$ & 4 & 3 \\
\hline & $40-49$ & 6 & 1 \\
\hline & $50-60$ & 8 & 3 \\
\hline \multirow[t]{6}{*}{ Education } & No school / Illiterate & 0 & 1 \\
\hline & Primary school & 0 & 2 \\
\hline & Secondary school & 0 & 2 \\
\hline & Technical high school & 9 & 1 \\
\hline & College & 4 & 1 \\
\hline & Undergraduate & 5 & 0 \\
\hline \multirow[t]{2}{*}{ Place of work for practitioners } & Township & 9 & N/A \\
\hline & Village & 9 & N/A \\
\hline \multirow[t]{2}{*}{ Place of residence for patients } & Township & N/A & 1 \\
\hline & Village & N/A & 6 \\
\hline \multirow[t]{2}{*}{ Location } & Xiuning County, Huangshan Municipality & 9 & 2 \\
\hline & Yingquan District*, Fuyang Municipality & 9 & 5 \\
\hline
\end{tabular}

*Participants were from the rural areas of this district 
patients with fever to specialised COVID-19 clinics. One participant said that the patient amount has reduced two-thirds' [P7, township practitioner] in his clinic.

'Any changes? That should be the decrease in patients. Patient numbers [have] decreased because the authority required that [we] cannot see [patients with] fever.' [P5, village TCM practitioner]

'They [authorities] usually asked you to go to the hospital to see a doctor, and you were not allowed to visit the grassroots level. Also [we] didn't dare to see a doctor, you may even be taken to the No. 5 Hospital, or [higher-level hospitals in] Fuyang if you're coughing.' [P22, primary school, village, 46 years]

Practitioners had little knowledge and experience of COVID-19 management and felt that information about this was not relevant to them.

'The treatment is none of our business, the physical examination is also none of our business. We only need to know that transferring patients immediately if there is certain symptom.' [P2, village practitioner]

'We've not been trained about the treatment [of COVID-19]. We mainly focused on preventions and infection control, because [patients who may need to receive] treatment were all transferred. This is our focus; we mainly highlighted the awareness of control and prevention.' [P13, head of THC]

\section{Concerns and challenges faced by those delivering primary health care}

Practitioners were very worried about catching the virus at the beginning of the outbreak because they were working on the frontline and initially lacked personal protective equipment (PPE). They faced uncertainty about their exposure from patients in the community: 'you contacted too many people at that time and had no idea at all about who may carry the virus' [P17, township TCM practitioner]. Some practitioners worried about transmitting the virus to their family and chose to live at their clinics for a time.

'Such worry is absolutely there. When this disease first outbreak, we didn't even have a mask, but we had to go [to the frontline] when we were told. We needed to check body temperature for people who returned from other places. Once there is a confirmed case, we will also have to be isolated. How far can you be away from them when checking the temperature? At the beginning, the winter, the supplies are scarce, and the masks are limited.' [P9, village practitioner]
'Well, I lived at the clinic in that half month and never went back home, since those [people he checked body temperature] are all returned from Wuhan, I definitely cannot go back home. ... Yes, it is [for the sake of protecting family], even we don't care, we should protect our family, right?' [P4, village practitioner]

Practitioners described how COVID-19 and the related changes in regulations had made their work more difficult and stressful. Despite the decrease in patients, their workload had actually increased due to COVID-19 control and prevention work. PPE was initially lacking and then difficult to use: '[you] cannot walk around [in protective gown] and [it] will be very difficult even to use the toilet' [P16, township internal medicine physician].

'That's true that patient numbers decreased, but this thing [the epidemic made you] feel alarmed, so the burden from mental side surely increased.' [P9, village practitioner]

'Workload should increase, we definitely have a high workload due to COVID-19. To what extent? We need to work day and night to, first, follow-up, and another, check body temperature, of returned people [from high risk areas], the more returning people the higher your workload would be.' [P10, village practitioner]

One participant felt the work of reporting and transferring all patients with possible COVID-19 symptoms was not achievable and instead he just asked patients with fever to leave his clinic: 'No referral, which will bring a lot of troubles. Just ask him [patient with fever] to leave. Yes, there is a complicated set of procedures for referral.' [P5, village TCM practitioner].

VCs experienced financial pressures as patient numbers and fees reduced and they became more reliant on income from the state for provision of public health infection prevention and control services. VCs had to scrap expired medicines purchased in anticipation of the normal level of need, but no financial compensation was available. Practitioners believed that they could maintain their business under such pressure, although it was more difficult than before.

'There must be [impacts]. Since the patient number in our clinic decreased, it definitely has impact on us.' [P3, village practitioner]

'These types of medicine were bought with our own money, so will they [authorities] care about the 
expiration? They will not care about it at all. There is no single penny from subsidy at all, not to mention others. There is nothing, just as same as usual.' [P5, village TCM practitioner]

'Patients [number] did decrease, [but] we also worked for public health education and the state ensured the money for this, so our life is guaranteed.' [P11, village practitioner]

Diminished primary health care and antibiotic prescribing VCs were no longer able to treat patients with common infections after the introduction of epidemic control regulation. There was no substitution of face to face with remote consultations (by phone or communications app), which practitioners attributed to the fact that most of their patients were elderly and were not used to remote consultations and/or don't know how to use smartphones.

'As for fever among local people, you know, the kind of cold [that can easily cause fever] especially for children. We have more child patients here, and in fact, it is very normal for them to have a fever caused by, for example, respiratory tract infections or tonsillitis, right?' [P8, township practitioner]

'There is no consultation [through WeChat or phone]. Almost all patients here are old people; remote consultation is rare, most of them will directly visit our village clinic.' [P12, village practitioner]

COVID-19 specialist clinics were established in some township centres and most staff were transferred there for pre-diagnosis at the start of the epidemic: 'many of us worked for patients with fever and used those fever clinics a lot at that time [at the start of the epidemic] when they have not been closed' [P13, head of THC]. However, these regional clinics soon closed to COVID19 patents since they lacked the qualified staff and equipment required. Patients with respiratory symptoms or fever then had travel even further for treatment.

'It was set up at the beginning; however, when the authorities came for inspection, they found these fever clinics did not meet the standards and also not have enough staff. These fever clinics were then withdrawn with only the referral counter remaining.' [P1, head of THC]

Patients found it more difficult to access health care as a result of the pandemic. Patients were afraid of being infected with COVID-19 and most patients said they would avoid places they viewed as having a high transmission risk, like health institutions, as a precaution. Patients said they were no longer able to access treatment at township or village level and some said that it was even difficult to get to pharmacies to purchase medicine.

'If there's not a necessary thing, I definitely don't want to go to hospital. After all there are many patients. What if I was all right but then be infected just because I visited there, right?' [P19, technical high school, village, 50 years]

'Our township [level health institutions] did not treat [patients with fever], they even did not prescribe medicine to them. Our local [health institutions] did not take any examination on them; they were all transferred to Fuyang City.' [P23, illiterate, village, 51 years]

Practitioners reported that treatment with antibiotics, which are commonly prescribed for fever and cough, has decreased since epidemic restrictions dramatically reduced the number of patients being seen at these clinics. The local lockdown further brought barriers to accessing antibiotics in THCs and VCs.

'There are impacts. Generally, like Otitis Media, Bronchitis, Tonsillitis, they generally are infections. Once they [patients] get fever, the problem is that we cannot prescribe medicines, so antibiotic use surely is less.' [P4, village practitioner]

It [the local lockdown] must be inconvenient to see a doctor, it's even impossible to go out once I wanted to buy some medicine for my niece.' [P22, primary school, village, 46 years]

Practitioners also reported reductions in antibiotic intravenous drip treatment because of concerns about increased risk of catching COVID-19 when spending time or gathering at health institutions.

'Because some periods ago, it should say that these recent two or three months were better, especially two or three months ago, the number of patients dropped sharply and sometimes there were no patients. Medicine used in intravenous infusion were likely to be scrapped quite a lot, most of them were antibiotic.' [P8, township practitioner]

'Intravenous drips at clinics were not allowed because it would lead patients to gather together, so intravenous drips were stopped at that time.' [P15, township practitioner] 


\section{COVID-19 as a different class of illness}

Practitioners described COVID-19 as different from the RTIs that, pre-pandemic, they had often treated in village and township clinics. This difference was characterised in a range of ways by practitioners. Many described it as much more serious. Some described it as having a different mode of action on the body or lungs. TCM practitioners understood COVID-19 through plague theory, the TCM theory used to explain illnesses with characteristics of high transmissibility and severity, rather than the 'hot-natured state' and 'hot' conditions that have been commonly associated with RTIs: 'In terms of TCM, COVID-19 is a plague [Wenyi] that related to outside environment' [P17, township TCM practitioner].

'It [COVID-19] is also inflammation, but its inflammation came quite fiercely and fast.' [P11, village practitioner]

'This COVID-19 will quickly cause shock and exhaustion; it's so severe. If you have general pneumonia, you can recover after admission in the hospital for ten or eight days, but COVID-19 is so severe and cannot be treated by common medicine.' [P11, village practitioner]

'[COVID-19 is] lung consolidation that is caused by the virus rather than inflammation' [P12, village practitioner]

COVID-19 was associated with SARS, also a serious epidemic that was not treated in the routine work of THCs and VCs. Like SARS, COVID-19 was thought to have high transmissibility and severity characteristics that were not commonly associated with normal RTIs and were of particular concern to practitioners and patients. COVID-19 was seen as more severe than SARS, partly due to the more severe and prolonged restrictions imposed to control it.

'Like SARS and COVID-19, they all belonged to corona virus, SARS in 2003 also came fiercely and also could lead to death. ... Our respiratory tract would also locate many viruses, but generally they do not cause such serious consequences, but this virus [COVID-19] is a big issue.' [P13, head of THC]

'[COVID-19 and] general pneumonia still have certain differences. First of all, for general pneumonia, it is not contagious in general situation. Look at it [COVID-19], then it is quite contagious; also, if there are some underlying conditions [patients] can be more likely to get severely ill from COVID-19, such as multiple organ failure.' [P15, township practitioner]
'Yes, this disease is more serious than SARS. SARS can be controlled. See how many days have been spent to control this disease? Isn't it still there?' [P21, college, town, 31 years]

'For sure, people will definitely be worried and afraid. In the past, even for SARS period, you didn't close the road, right? Now the villages are closed, you are not allowed to go out, and then the roads are closed, some places the roads are even cut off so you cannot use them and go out. It feels more serious.' [P25, secondary school, village, 35 years]

\section{The impact of COVID-19 on understandings of antibiotic} use

Practitioners believed that the COVID-19 epidemic had not led to any changes in their antibiotic treatment practices, except that they were seeing lower numbers of patients presenting with infections and therefore the amount of antibiotics prescribed had decreased during the epidemic period. Practitioners' views on how and when antibiotics should be used were found to be the same as before the epidemic. Some of them said that the use of antibiotics was decreasing irrespective of the Covid-19 pandemic due to gradually strengthened measures specifically to control antibiotic use over recent years.

'That is to say, for COVID-19 epidemic's own impact on the daily use of antibiotics and the consultation, I don't think it is much different from previous years.' [P8, township practitioner]

'Our diagnosis and treatment will continue as before. The use of antibiotics will still be strictly [controlled]. Early-stage colds will not recommend using antibiotics, [they] are all anti-viral treatment. Antibiotics should not be used until obvious infections like tonsillitis.' [P6, village practitioner]

Since practitioners viewed COVID-19 as different to the common RTIs seen in their daily work, they did not see their treatment experiences as relevant to COVID-19 or information about COVID-19 treatment as relevant to their practice.

'Village clinics would not be interested in that, doctors in village clinics are impossible to see how antibiotics could be used for COVID-19; they won't even look at it' [P4, village practitioner]

Patients did not make any connections between antibiotics and COVID-19 and did not report having used antibiotics to prevent or treat COVID-19. Their views on antibiotics were unchanged by the epidemic: patients 
believed that antibiotics reduced the duration of illness and said it was common practice both to seek antibiotics from clinics, and to self-medicate for infections with antibiotics purchased from retail pharmacies without a prescription. Practitioners similarly believed that COVID-19 would not affect patients' antibiotic use practices and that public understanding of antibiotics was limited.

'I have no idea if anti-inflammatory drugs [antibiotics] will be useful for COVID-19. Researcher: So have you ever used antibiotics to prevent COVID19? P24: Not ever used.' [P24, secondary school, village, 54 years]

'Sometimes just asked them [retail pharmacies] to give some anti-inflammatory drugs [antibiotics], they can [help illness] to recover quicker.' [P19, technical high school, village, 50 years]

'I feel that public's understanding should be that they [antibiotics and COVID-19] don't have any relationship, the public would not link these two together. Yes, they will not believe that, that antibiotics are related to COVID-19, right.' [P15, township practitioner]

\section{Discussion}

Our study identified a shift in PHC workload and responsibility to epidemic control and prevention in rural areas of central China over the first phase of COVID-19 epidemic. Pre-pandemic, PHC treated many patients with RTIs, but as part of epidemic control procedures PHC institutions were not allowed to diagnose or treat any infections with suspected COVID-19 symptoms, and were not familiar with COVID-19 management, which was designated to specialist facilities. Both practitioners and patients reported a decrease in $\mathrm{PHC}$ delivered at community level. Practitioners were concerned about catching the virus, increased work pressure, and financial pressure, particularly in VCs. Antibiotic prescribing decreased in THCs and VCs because of the reduction in numbers of patients being seen at these clinics. However, participants believed that COVID-19 had not changed antibiotic related practices, since participants perceived COVID-19 as different from common RTIs and therefore not relevant to their normal treatment of RTIs.

This study shows the key role played by rural PHC practitioners in COVID-19 screening and monitoring. In response to the epidemic, these PHC services considerably increased their public health work (tracing, screening, education), while their provision of general medical services was reduced because patients were referred elsewhere or avoided clinics. This study did not find the shift to remote consulting that has been observed in
PHC systems in countries such as the UK [2]. China's national guidelines also did not make any recommendations about switching to remote consultations to maintain essential clinical services [16]. Since most rural residents are elders [31], remote consultations would be difficult to conduct in rural areas in China since older residents are reluctant or unable to forego the normal face to face consultation.

The reassignment of PHC practitioners away from patient treatment and into public health tracing, referral and educational roles highlights the challenge of a PHC workforce with limited medical training. The quality of diagnosis and treatment at rural PHC has been evaluated to be low [32], with a high proportion of inadequately trained practitioners [10]. The most well-qualified health practitioners tend to be concentrated in large hospitals and urban areas, contributing to inequities between rural and urban areas in terms of access to better qualified health practitioners $[10,33]$. For patients, the pandemicrelated avoidance of $\mathrm{PHC}$ institutions and the additional travel required to access treatment if referred reduce access to health care and essential medicine, particularly in rural areas where over $80 \%$ of first-time care previously occurred in primary care [14].

The COVID-19 epidemic brought considerable additional pressures and stress for practitioners, particularly those working in VCs. There were widespread concerns about catching the virus and PPE shortages across the entire health care system in China [17, 34], as well as among health workers in other countries [35]. Work pressures and fatigue from the high workload related to paperwork and reporting were described from practitioners elsewhere in China's PHC system, and it was suggested that additional staff were needed for these low-skilled tasks so that practitioners could focus more on diagnosis and treatment [17]. Our study indicated that, compared with THCs, the reduction of patient numbers and fees caused more obvious financial pressures on VCs. This is related to their financially semi-autonomous status that means the clinic's overheads, operational costs, and the majority of the staff salaries need to be covered by income generated from seeing and treating patients [12]. These financial considerations have been identified as a critical reason for 'village doctors' to prescribe antibiotics, including intravenous administration which generates more income [14], since they are linked to the economic survival of practitioner and clinic. However, practitioners participating in this study reported that their VCs were able to maintain their business despite substantial decreases in patients attending and a corresponding drop in antibiotic prescriptions, suggesting the possibility that clinics may be economically viable without the overuse of antibiotics. 
Participants in our study reported a decrease in antibiotic prescribing at the rural community level during COVID-19. In the UK, there was a reduction of $15 \%$ in the number of antibiotic prescriptions issued in 2020, compared with the corresponding period in 2019 [36]. As with our study, the reduction in antibiotic prescriptions in UK primary care was the result of a decrease in the absolute number of appointments, however the rate of antibiotic prescriptions per patients actually increased, which was linked to greater diagnostic uncertainty from remote consultations [36].

Practitioners and patients perceived COVID-19 to be a very different type of illness to other RTIs. This was despite the apparent similarity in symptoms with fever and cough being key indicators of suspected COVID-19, of common RTIs and, pre-pandemic, frequently taken to indicate an infection/'inflammation' that needed antibiotic treatment [14]. COVID-19 was associated with SARS because of the similarity in the response of the health care system and national control measures. SARS differed from previous infectious diseases in the rapid spread that hit China's health care system and caused widespread panic [37, 38]. The national regulations and guidelines on specialised referral and treatment process further emphasised COVID-19 as a unique class of illness. The severity of COVID-19, including characteristics of rapid development, high transmissibility and fatality, are in line with TCM plague (wenyi) theory, which assigns COVID-19 to the category of plagues with different properties from other illnesses in TCM, including general RTIs [39, 40]. In TCM aetiology, the cause of COVID-19 is epidemic miasma (liqi), which is different to the six excesses (liuyin) (including excesses of wind, cold, summer heat, dampness, dryness, fire) that are believed to cause common cold and fever [41]. COVID-19 was understood by participants as an entirely different types of illness from the RTIs the practitioners commonly diagnosed and treated in their clinics and therefore knowledge of COVID-19 was not seen as relevant to their practice.

Our qualitative study has provided new and detailed evidence of the impact of COVID-19 on PHC system and antibiotic prescribing in rural China. The study recruited practitioners and patients from both high and low incidence areas and included a diverse range of rural practitioners from THCs and VCs in various specialities. The number of patients participating in this study was small, but their views added an important perspective on the changes to health care services described by practitioners. The community pharmacy, which is an important source for rural residents to access essential medicines and antibiotics, was not included in our study since its responsibilities in response to COVID-19 was more limited. To understand COVID-19's impact on the use of antibiotics in the community, future studies should include pharmacy staff and customers.

\section{Conclusions}

The COVID-19 epidemic has had a considerable impact on the delivery of $\mathrm{PHC}$ in rural China. PHC practitioners took on the key public health role of tracing, screening and educating in rural areas, while their role in seeing and treating patients was reduced, since many patients were diverted to specialist COVID-19 clinics. There was no transition to remote consultations, which was partly attributed to the diversion of patients away from less well trained rural practitioners towards more qualified medical practitioners in urban areas, and partly to high numbers of elderly patients in rural areas who had neither technology nor inclination for remote consultations. The additional work and risk that $\mathrm{PHC}$ practitioners faced placed considerable strain on them, particularly those working in the VCs.

Since local COVID-19 outbreak and epidemic control work has been designated as a long-term task in China, rural PHC clinics now face the challenge of how to balance their principal clinical and public health roles and, in the case of the VCs, remain financially viable. Support from non-medical staff or community workers to help with the non-clinical work associated with epidemic control, such as recording and reporting, could reduce practitioners' workload and enable them to focus more on diagnosis and treatment. Improved local PHC provision providing access to basic medical services is needed, particularly by those local residents who are old or cannot afford the additional travel. Continuing national efforts to strengthen the rural PHC system and to improve the quality of training of $\mathrm{PHC}$ practitioners are needed to enable them to carry out these key dual responsibilities of front line medical care and epidemic control work. With respect to the national drive to reduce the overuse of antibiotics, we found evidence that VCs could maintain their business despite substantial decreases in antibiotic prescriptions; exploring strategies to help VCs develop plans for economic sustainability, while reducing their reliance on income linked to prescribing antibiotics, would help promote antimicrobial stewardship in VCs.

\section{Abbreviations}

PHC: Primary health care; PPE: Personal protective equipment;

RTI: Respiratory tract infection; SARS: Severe acute respiratory syndrome; TCM: Traditional Chinese medicine; THC: Township health centre; UTI: Urinary tract infection; VC: village clinic 


\section{Supplementary information}

The online version contains supplementary material available at https://doi. org/10.1186/s12913-021-07082-z.

\section{Additional file 1 \\ Additional file 2 \\ Additional file 3}

\section{Acknowledgements}

We are grateful to all MSc students in Anhui Medical University for their transcribing work and to senior staff in local PHC who helped with the recruitment. Many thanks to participants who contributed to this study.

\section{Authors' contributions}

All authors conceptualized and designed the study. TZ, XS, RL and LZ were involved in recruitment and data collection, TZ and CC were involved in data analysis and interpretation. TZ led the drafting of the manuscript. Al authors contributed to, and read and approved, the final version of the manuscript.

\section{Funding}

The authors acknowledge UK Medical Research Council (MRC) \& Newton Fund through a UK-China AMR Partnership Hub award (MR/S013717/1), and the National Natural Science Foundation of China (NSFC) award (81661138001).

\section{Availability of data and materials}

The datasets generated and analysed during the current study are not publicly available due to raw interview transcripts when considered as a whole may potentially be identifying. Data supported findings in this study have been provided in the paper. The datasets in the current study are available from the corresponding author on reasonable request.

\section{Declarations}

\section{Ethics approval and consent to participate}

This study has been reviewed and approved by the Ethics Committee of Anhui Medical University (reference number 2020H0110). All methods were performed in accordance with the relevant guidelines and regulations. Audio recorded verbal consent has been obtained from all participants and it is approved by the Ethics Committee.

\section{Consent for publication}

Not applicable.

\section{Competing interests}

The authors declare that they have no competing interests.

\section{Author details}

${ }^{1}$ Population Health Sciences, Bristol Medical School, University of Bristol, Bristol, UK. ${ }^{2}$ School of Health Services Management, Anhui Medical University, Hefei, China. ${ }^{3}$ Centre for Academic Primary Care, Population Health Sciences, Bristol Medical School, University of Bristol, Bristol, UK.

Received: 19 May 2021 Accepted: 21 September 2021

Published online: 05 October 2021

\section{References}

1. Kidd M. Australia's primary care COVID-19 response. Aust J Gen Pract 2020;49 Suppl 2.

2. Murphy M, Turner A, Denholm R, et al. RAPCI Project Summary Report 4, 31 July 2020. Centre for Academic Primary Care (CAPC), University of Bristol.

3. Department of Primary Health of National Health Commission (DPHNHC). Primary care institutions' key role in epidemic control. DPHNHC 2020a. [Online] Available at: http://www.nhc.gov.cn/jws/s3578/202002/19c2e477e8c04e2 0b76730daeb1296f8.shtml [Accessed 08 Jan 2021]

4. DPHNHC. National Health Commission Office notification on strengthening COVID-19 prevention and control work among primary care institutions. DPHN
HC 2020b. [Online] Available at: http://www.nhc.gov.cn/jws/s7874/202001/ 0165523421f840af816a580f260d4406.shtml [Access 08 Jan 2021]

5. Li X, Krumholz H, Yip W, et al. Quality of primary health care in China: challenges and recommendations. Lancet 2020;395:1802-12.

6. DPHNHC. Fighting against the epidemic: the actions of local primary care practitioners 1. DPHNHC 2020c. [Online] Available at: http://www.nhc.gov.cn/ jws/s7873/202002/1e9e03e8a881408d848192d66f4c3622.shtml [Accessed 08 Jan 2021]

7. DPHNHC. Fighting against the epidemic: the actions of local primary care practitioners 3. DPHNHC 2020d [Online] Available at: http://www.nhc.gov.cn/ jws/s7873/202002/426eb41e86b645e4a0f9a589399ced54.shtml [Accessed 08 $\operatorname{Jan} 2021]$

8. DPHNHC. Fighting against the epidemic: the actions of local primary care practitioners 2. DPHNHC 2020e. [Online] Available at: http://www.nhc.gov. cn/jws/s7873/202002/41a0250ddc5d4d1bb6fe721ad6a00201.shtml [Accessed 08 Jan 2021]

9. World Health Organization (WHO). Regional Office for the Western Pacific People's Republic of China health system review. Manila: WHO Regional Office for the Western Pacific 2015

10. Li X, Lu J, Hu S, et al. The primary health-care system in China. Lancet 2017; 390:2584-94.

11. Hu D, Zhu W, Fu Y, et al. Development of village doctors in China: financial compensation and health system support. Int J Equity Health 2017;16:9.

12. Chen M, Kadetz P, Cabral C, et al. Prescribing antibiotics in rural China: the influence of capital on clinical realities. Front Sociol 2020;5.

13. Dong L, Yan H \& Wang D. Drug prescribing indicators in village health clinics across 10 provinces of Western China. Fam Pract 2011;28:63-67.

14. Lambert $H$, Chen M, Cabral C. Antimicrobial resistance, inflammatory responses: a comparative analysis of pathogenicities, knowledge hybrids and the semantics of antibiotic use. Palgrave Commun 2019;5.

15. National Health Commission (NHC). 2019 Annual China Health Statistics. Beijing: China Xiehe Medical University publications 2019: Sect. 5.14.2.

16. Yin $X$, Song $F$, Gong $Y$, et al. A systematic review of antibiotic utilization in China. J Antimicrob Chemother 2013:68:2445-52.

17. WHO. Using Indicators to Measure Country Pharmaceutical Situations: Fact Book on WHO Level 1 and Level 11 Monitoring Indicators. Geneva: World Health Organisation 2006.

18. Haldane V, Zhang Z, Abbas RF, et al. National primary care responses to COVID-19: a rapid review of the literature. BMJ Open 2020;10:e041622.

19. $X u Z$, , Ye $Y$, Wang $Y$, et al. Primary care practitioner' barriers to and experience of COVID-19 epidemic control in China: a qualitative study. $J$ Gen Intern Med 2020;35:3278-84.

20. Zhao L, Kwiatkowska RM, Chai J, et al. Pathways to optimising antibiotic use in rural China: identifying key determinants in community and clinical settings, a mixed methods study protocol. BMJ Open 2019:9:e027819.

21. Baidu. The report of real-time data on the COVID-19 epidemic. Baidu 2020. [Online] Available at: https://voice.baidu.com/act/newpneumonia/ newpneumonia/?from=osari_aladin_banner\&city=\%E5 \%AE\%89\%E5 \%BE\% BD-\%E5 \%AE\%89 \%E5 \%BE\%BD [Accessed 25 Nov 2020]

22. Cong W, Chai J, Zhao L, et al. A cluster randomised controlled trial to assess a tailored intervention to reduce antibiotic prescribing in rural China Study protocol. BMJ Open 2021. Under review.

23. Huang Y, Pan S. Research ethics in social surveys in China: methodological reflections. Soc Sci China 2009;2:149-62.

24. Liu J. Researching Chinese women's lives: 'insider' research and life history interviewing. Oral Hist 2006;34:43-52.

25. Liu J. Gender and Work in Under China: Women Workers of the Unlucky Generation. London: Routledge 2007.

26. Gobo G. Glocalizing methodology? The encounter between local methodologies. Int I Soc Res Methodol 2011;14:417-37.

27. Snape D \& Spencer L. The Foundation of Qualitative Research. In: Ritchie J \& Lewis I (eds.). Qualitative Research Practice: A Guide for Social Science Students and Researchers. London; Thousand Oaks; New Delhi: Sage Publications 2003.

28. Holloway I. A-Z of Qualitative Research in Healthcare. 2nd edn. Oxford: Blackwell Publication 2008.

29. Guest G, Bunce A, Johnson L. How Many Interviews Are Enough?: An Experiment with Data Saturation and Variability. Field Methods 2006;18:59-82.

30. Braun V \& Clarke V. Chapter 4 Thematic analysis. In: Cooper H (editor-inchief). APA Handbook of Research Methods in Psychology: Vol. 2. Research Designs. American Psychological Association 2012. 
31. Dai B, Zhou J, Mei Y, et al. Can the New Cooperative Medical Scheme promote rural elders' access to health-care services?. Geriatr Gerontol Int 2011;11:239-45.

32. Su M, Zhang Q, Bai X, et al. Availability, cost, and prescription patterns of antihypertensive medications in primary health care in China: A nationwide cross-sectional survey. Lancet 2017;390:2559-68.

33. Hou J, Ke Y. Addressing the Shortage of Health Professionals in Rural China: Issues and Progress; Comment on 'Have Health Human Resources Become More Equal between Rural and Urban Areas after the New Reform?'. Int J Health Policy Manag 2015;4:327-28.

34. Liu Q, Luo D, Haase JE, et al. The experiences of health-care providers during the COVID-19 crisis in China: a qualitative study. Lancet Glob Health 2020;8:e790-98.

35. Sterling MR, Tseng E, Poon A, et al. Experience of home health care workers in New York City during the coronavirus disease 2019 pandemic A qualitative analysis. JAMA Intern Med 2020;180:1453-59.

36. Armitage $R$ \& Nellums LB. Antibiotic prescribing in general practice during COVID-19. Lancet Infect Dis Correspondence 2021;21:E144.

37. Hung LS. The SARS epidemic in Hong Kong: what lessons have we learned?. J R Soc Med 2003;96:374-8.

38. Zhong $\mathrm{N}$, Zeng G. What we have learnt from SARS epidemics in China. BMJ 2006:333:389-91.

39. Yin H, Zhang B. Traditional Chinese Medicine Basic Sciences. Shanghai: Shanghai Science and Technology Publication 2006.

40. Zhao Z, Li Y, Zhou L, et al. Prevention and treatment of COVID-19 using Traditional Chinese Medicine: A review. Phytomedicine 2020

41. Li J, Li J, Liang T, et al. Analysis of Novel Coronavirus Pneumonia syndrome characteristics and prevention based on the Traditional Chinese Medicine Plague Theory. World J Tradit Chin Med 2020;15:172-76.

\section{Publisher's Note}

Springer Nature remains neutral with regard to jurisdictional claims in published maps and institutional affiliations.

Ready to submit your research? Choose BMC and benefit from:

- fast, convenient online submission

- thorough peer review by experienced researchers in your field

- rapid publication on acceptance

- support for research data, including large and complex data types

- gold Open Access which fosters wider collaboration and increased citations

- maximum visibility for your research: over $100 \mathrm{M}$ website views per year

At $\mathrm{BMC}$, research is always in progress.

Learn more biomedcentral.com/submissions 